Motrivivência Ano XXV, No 40, P. 67-79 Jun./2013

http://dx.doi.org/10.5007/2175-8042.2013v25n40p67

\title{
AVALIAÇÃO DA ORIENTAÇÃO MOTIVACIONAL DE METAS NA MODALIDADE DO FUTEBOL
}

\author{
Vinicius Barroso Hirota' \\ Ademir De Marco ${ }^{2}$ \\ Carlos Eduardo Lopes Verardi ${ }^{3}$ \\ Reginaldo de Moura Gomes ${ }^{4}$ \\ Elias De França ${ }^{5}$
}

\section{RESUMO}

O presente trabalho objetivou avaliar e comparar a orientação motivacional de meta para tarefa e ego de alunos pertencentes a dois ambientes esportivos distintos de futebol, uma Escola de Esporte Privada e uma Organização Não Governamental (ONG), ambos localizados na Cidade de Osasco - SP. Através de uma pesquisa descritiva aplicamos a escala TEOSQ, a uma amostra de 196 sujeitos de ambos os sexos de idade entre 7 e 17 anos (média 11,16+2,09) e os dados foram tratados com o calculo do Coeficiente Alfa, média, desvio padrão, mediana e comparadas as médias com o teste de Mann Whitney. Os resultados mostraram estabilidade no instrumento com Alfa de 0,78 para tarefa e 0,68 de ego na escola de esporte com média de 4,28 de tarefa e 2,40 de ego, já a ONG o Alfa de tarefa foi 0,60 e 0,74 de ego com média de tarefa de 4,40 e ego 2,75 . Concluímos a tendência de orientação para tarefa com alunos predispostos a se superarem constantemente, e a fidedignidade do instrumento

Palavras-chave: Avaliação; Futebol; Motivação; Orientação de Metas.

1 Mestre em Educação Física. Universidade Presbiteriana Mackenzie. Consolação/São Paulo, Brasil. E-mail: vhirota@mackenzie.com.br.

2 Doutor em Anatomia/Neuroanatomia Humana. Universidade Estadual de Campinas. Campinas/São Paulo, Brasil. E-mail: demarco@fef.unicamp.br.

3 Doutor em Ciências da Saúde. Universidade Estadual Paulista. Baurú/São Paulo, Brasil.

E-mail: verardi@fc.unesp.br.

4 Bacharel em Educação Física. Associação Esporte Solidário. São Paulo/São Paulo, Brasil.

E-mail: regismgsr@hotmail.com.

5 Graduando do curso de Educação Física. Universidade Presbiteriana Mackenzie/Bolsista CNPq. Consolação/ São Paulo, Brasil. E-mail: elias.dqe.qf@hotmail.com. 


\section{INTRODUÇÃO}

A motivação no contexto esportivo se faz necessária para a aprendizagem e aperfeiçoamento da técnica, tática e desempenho físico do jogador, além da busca de sua auto superação. O futebol é um esporte muito visto no cenário nacional, porém o que muitos jovens não sabem é que no futebol é imprescindível ter uma motivação marcante para a sua pratica.

Verardi (2004) fala que a partir dos aspectos emocionais, procura - se fazer uma relação entre a ação e a vivência esportiva, tanto no seu aspecto competitivo quanto na prática do futebol, presentes na experiência.

Gomes, Santos, Hirota e De Marco (2011) afirma que a motivação no contexto esportivo se faz necessária para a aprendizagem e aperfeiçoamento da técnica, tática e desempenho físico dos jogadores de futebol durante uma partida e um treinamento esportivo.

Weinberg e Gould (2001) relatam que a motivação é utilizada como uma força, podendo fazer com que o individuo possa realizar uma ação ou a pratica de um determinado esporte, com o direcionamento dessa motivação. Já para alguns psicólogos do esporte ela pode ser vista de diferentes maneiras como de estresse competitivo onde o atleta pode ficar nervoso, ansioso, preocupado entre outros, também pode ser visto como uma motivação intrínseca para o atleta se alto motivar, com essa motivação partindo do próprio atleta, ou extrínseca onde o ambiente pode sofrer alterações e o atleta pode sentir-se motivado ou não, com isso, o atleta poderá ser motivado de diferentes maneiras para um melhor desempenho.
Dentro ainda da busca de definições para motivação, Murray (1983) afirma que um motivo é um fator interno que dá início, dirige e integra o comportamento de uma pessoa.

Thomas (1983 citado De Marco e Junqueira, 1995) diz que motivos não conduzem a ação. São situações em um determinado momento que despertam e estimulam motivos de modo a conduzi - lós efetivamente à ação.

Winterstein (1992) classifica a teoria da motivação partindo do pressuposto de que deve existir alguma coisa que desencadeia uma ação, que Ihe dá uma direção, mantém seu curso em direção a um objetivo e a finaliza. O mesmo autor completa esclarecendo, que motivos são construções hipotéticas, que são aprendidas ao longo do desenvolvimento humano e servem para explicar comportamento.

Desta maneira a motivação do individuo pode melhorar ou piorar os seus desempenhos no esporte, mais também orienta para que ao realizarem suas pretensões, para persistirem quando errar e sentir orgulho quando atingir os seus objetivos (Winterstein, 2002).

Segundo Magill (1984) a motivação é importante para a compreensão da aprendizagem e do aprendizado de habilidades motoras devido a seu papel na iniciação, manutenção e intensidade do comportamento.

Paim (2001) nos diz que a motivação é muito importante para se buscar qualquer objetivo pelo ser humano, aonde a motivação serve como um elemento básico para esse atleta seguir orientações de seus treinadores e técnicos, e poder praticar diariamente as suas sessões de treinos.

Gaya e Cardoso (1998) relatam que os motivos na qual definem as atividades desportivas são o melhoramento das 
habilidades a vivência de emoções, passarem bem, e o bem estar do individuo, entre outros fazendo com que o individuo passe a ter um progresso pessoal aonde ela irá determinar suas conquistas pessoais.

Portanto a motivação pode ser descrita como a força que direciona ou não um individuo a fazer uma ação ou a praticar determinado esporte. Para alguns psicólogos do esporte ela pode ser vista de diferentes ângulos, na forma de estresse competitivo ou realização e motivação intrínseca e extrínseca, todas formando a definição geral de motivação (Weimberg \& Gould, 2001).

Weinberg e Gould (2001) dizem que ao correlacionarem à motivação intrínseca e recompensas externas, afirmam que as recompensas extrínsecas têm o potencial de abalar a motivação intrínseca.

Winterstein (2002) remete esta teoria á visão da expectativa de êxito e medo do fracasso, ou seja, a expectativa de êxito apresenta semelhanças com indivíduos orientados para tarefa (Nicholls, 1984) e medo do fracasso assemelha - se a indivíduos orientados para o ego (Nicholls, 1984). Ainda Winterstein (2002) relata que a expectativa ou a probabilidade (de êxito ou de fracasso) diz respeito à probabilidade de êxito que o indivíduo estabelece. Ela desenvolve - se a partir de experiências passadas em situações semelhantes que o sujeito enfrentou.

Hirota, De Marco e Verardi (2009) relatam que os indivíduos que possuem uma auto referencia as habilidades atribuem o sucesso para equipe, juntamente ao esforço e a determinação das habilidades.

Sendo assim Chaves (2002) diz que segundo a teoria cognitiva da motivação, o comportamento é dirigido pela possibilidade de antecipar os fins a serem atingidos e os aspectos internos são valorizados, como por exemplo objetivos, intenções, expectativas e planos individuais; o individuo pode decidir conscientemente o que quer ou não fazer, de acordo com sua satisfação e necessidade.

Duda (1992) diz que existe um elo entre a orientação motivacional de um indivíduo em determinado ambiente particular e a socialização potencial de valores inerentes neste ambiente. Assim quando falamos que um indivíduo tem orientação para ego algumas características são ressaltadas como individualismo e tarefas com menor grau de dificuldade e aquele indivíduo orientado para tarefa possui algumas características como uma auto referência em relação às habilidades, espírito de equipe e cooperação.

Por isso a importância do desenvolvimento desta pesquisa é voltada para identificação, avaliação e comparação da orientação motivacional de jovens praticantes de futebol observando a necessidade da motivação na melhora e/ ou manutenção do desempenhando esportivo no contexto do futebol.

\section{OBJETIVO}

Avaliar e comparar a orientação motivacional de metas de alunos pertencentes a dois ambientes esportivos distintos:

a. Uma escola de futebol particular;

b. Projeto social de escola de esporte de futebol, Organização Não Governamental (ONG).

\section{Objetivos específicos}

1. Testar a validade e fidedignidade do instrumento; 
2. Determinar as características de cada orientação motivacional (meta Tarefa e meta Ego), separadamente por níveis de desenvolvimento proposto por Freire (2003), de cada estabelecimento esportivo;

3. Avaliar a existência de possíveis diferenças significativas entre as orientações motivacionais (meta Tarefa e meta Ego) de cada estabelecimento esportivo, bem como os diferentes níveis de desenvolvimento proposto por Freire (2003);

4. Evidenciar se existe diferença significativa separadamente de cada orientação motivacional (meta Tarefa e meta Ego) entre os estabelecimentos esportivos.

\section{METODOLOGIA}

\section{Modalidade da Pesquisa}

Este estudo se caracterizou como uma Pesquisa de Campo, do Tipo descritiva, na qual conforme Marconi e Lakatos (2002) consistem em uma investigação de pesquisa empírica cujo fator principal é o teste de hipóteses que correspondem à relação de tipo causa - efeito.

\section{Local de Estudo e Amostra}

A amostra foi constituída por dois locais distintos, uma escola de esporte e uma ONG ambos situados na Cidade de Osasco, SP, contando com a participação total de 196 sujeitos, dos quais 12 são do sexo feminino, e 184 do sexo masculino.
O primeiro local é uma escola de futebol particular, localizada em uma área comercial, que tem como intuito desenvolver especificamente esta modalidade, partindo do pressuposto das questões individuais dos sujeitos envolvidos no processo de ensino aprendizagem, a amostra de sujeitos deste local compreendeu 148 crianças (n: 148), do sexo masculino, faixas etárias entre 07 e 15 anos (média de idade 10,95 +2,04).

O segundo local de estudo é uma Organização Não Governamental (ONG), localizada em uma comunidade carente, onde os alunos e alunas estão envolvidos em diversas atividades (Jiu-Jítsu, Dança e Futebol) cujo objetivo da é promover o desenvolvimento social e motor dos alunos e contou com a participação de 48 (n: 48) indivíduos com idade entre 8 a 17 anos, no entanto neste local consideramos 36 meninos (n: 36, média de idade 11,4 $\pm 1,98$ ) e 12 meninas ( $\mathrm{n}: 12$, média de idade de 13,2 $\pm 2,12$ ) que participam das praticas desenvolvidas pelo projeto social em horários diferentes durante a semana.

Como único critério de inclusão, os participantes deveriam estar devidamente matriculados na modalidade inserida no contexto do futebol a mais de um mês, e os pais ou responsável estarem de acordo em assinarem o Termo de Consentimento Livre e Esclarecido.

\section{Instrumentação}

Para a realização deste trabalho foram entregues os questionários sobre aplicação de uma escala de identificação da orientação motivacional, TEOSQ (TASK AND EGO ORIENTATION IN SPORT QUESTIONAIRE) Questionário do Esporte 
de Orientação para Tarefa ou Ego desenvolvida por Duda (1992), traduzida, adaptada e validada por Hirota e De Marco (2006b) o qual nos permite identificar a orientação motivacional de metas, verificando se sua orientação estava dirigida para a meta tarefa ou para a meta ego; o questionário é composto por 13 questões sendo 07 questões para a tarefa e 06 questões para o ego, do tipo Likert, e as opções de resposta possíveis para cada uma variam de 1 a 5 pontos.

Com isso a finalidade do TEOSQ Questionário do Esporte de Orientação para Tarefa ou Ego - é a de avaliar as diferenças individuais em perspectiva do objetivo ajustado ao esporte, detectando se o indivíduo é pendente a ser orientado para tarefa ou orientado para ego.

\section{Coleta de Dados}

O estudo seguiu os preceitos da ética em pesquisa, de acordo com a Resolução n. 196/ 96 do Conselho Nacional de Saúde, após a leitura e assinatura dos Termos de Consentimento das Instituições, primeiro pelo responsável da instituição, a próxima etapa foi a assinatura do Termo de Consentimento Livre e Esclarecido junto aos pais ou responsável, uma vez que os alunos compreendem idade de 07 a 17 anos.

A aplicação do instrumento ocorreu nas instalações de ambas as instituições, com a presença de um responsável de cada instituição, não havendo nenhum gasto adicional para os sujeitos, voluntários da pesquisa.

Analise dos Dados

Adotamos como tratamento estatístico o cálculo do Coeficiente Alfa de
Cronbach a fim de verificar a validade e fidedignidade da escala, colocando a prova a validade do instrumento frente aos alunos praticantes de futebol. Este é um coeficiente de fidedignidade generalizado que é mais versátil do que os outros métodos e uma característica deste coeficiente é que pode ser utilizado com itens que tem várias medidas de valores, tais como teste de redação e escalas de atitudes com pontuação como concordo fortemente, concordo etc. Além disso, o Alfa é provavelmente o melhor coeficiente para estimar a fidedignidade mais comumente usada nos testes padronizados (Thomas \& Nelson, 2002).

Cálculos também as médias, desvio padrão e mediana de cada orientação, ou seja, apresentamos resultados das orientações de meta tarefa e também meta de ego, separadamente de acordo com a classificação dos níveis de desenvolvimento do futebol proposto por Freire (2003), onde os alunos de 7 a 9 anos se enquadram no nível de desenvolvimento Iniciante, os alunos de 10 e 13 anos no nível Básico, e os alunos de 14 a 17 anos no Especial, este último a participação dos alunos em competições torna-se mais frequente.

Por fim para assistir se existe uma diferença significativa entre as médias das metas de Tarefa e Ego, optamos por realizar o teste de Mann Whitney com nível de significância de $p<0,05$. Executamos um teste de One Way Anova (pós teste de Tukey), separadamente para cada orientação, ou seja, orientação para meta tarefa e orientação para meta ego, ressalvando a existência, ou não, de diferenças significativas entre os níveis de desenvolvimento Iniciante, Básico e Especial.

Contamos com o emprego do software SPSS - DATA EDITOR, versão 17.0 for Windows para as aferições. 


\section{RESULTADOS E DISCUSSÃO}

A primeira testagem estabelecida validade da escala TEOSQ, visualizando a sua fidedignidade através do calculo do Coeficiente Alfa de Cronbach. pelo objetivo do estudo foi verificar a

Tabela 01: Calculo do Coeficiente Alfa de Orientação de Tarefa e de Ego da Escola de Esporte.

\begin{tabular}{lcccc}
\hline $\begin{array}{l}\text { Nível de } \\
\text { desenvolvimento }\end{array}$ & Idade & $\mathbf{n}$ & $\begin{array}{c}\text { Alfa } \\
\text { ego }\end{array}$ & $\begin{array}{c}\text { Alfa } \\
\text { tarefa }\end{array}$ \\
\hline Iniciante & 7 a 9 & 37 & 0.61 & 0.80 \\
Básico & 10 a 13 & 89 & 0.70 & 0.74 \\
Especial & 14 a 17 & 22 & 0.73 & 0.85 \\
\hline Total & 7 a 17 & 148 & 0.68 & 0.78 \\
\hline
\end{tabular}

Analisando os dados da Tabela 01, referente à escola de esportes obtivemos resultados satisfatórios em relação tanto a orientação para tarefa como para ego, demonstrando assim a estabilidade do instrumento.

Os mesmos resultados podem ser encontrados na Tabela 02 em relação à ONG, no entanto apesar de satisfatório, podemos observar que o existiu pouco entendimento nas questões referente a orientação para a tarefa, uma vez que o resultado de Alfa ficou aquém de outros estudos. Vale lembrar que este resultado pode variar de 0 a 1 , e quanto mais próximo de 1 melhor o resultado obtido.

Para a ONG podemos dizer que as questões para a tarefa não foram interpretadas da melhor maneira nas idades dos níveis básico e especial.

Tabela 02: Calculo do Coeficiente Alfa de Orientação de Tarefa e de Ego da ONG.

\begin{tabular}{lcccc}
\hline $\begin{array}{l}\text { Nível de } \\
\text { desenvolvimento }\end{array}$ & Idade & $\mathbf{n}$ & $\begin{array}{c}\text { Alfa } \\
\text { ego }\end{array}$ & $\begin{array}{c}\text { Alfa } \\
\text { tarefa }\end{array}$ \\
\hline Iniciante & 7 a 9 & 7 & 0.68 & 0.71 \\
Básico & 10 a 13 & 32 & 0.72 & 0.57 \\
Especial & 14 a 17 & 9 & 0.84 & 0.52 \\
\hline Total & 7 a 17 & 48 & 0.74 & 0.60 \\
\hline
\end{tabular}

Este instrumento tem sido empregado e estatado em indivíduos na infância e na adolescência, em jovens e adultos do sexo masculino e feminino praticantes do futebol (Hirota, Schindler, \& Villar, 2006a; Hirota e Tragueta, 2007; Camargo, Hirota,
\& Verardi, 2008; Hirota et al., 2009; Hirota, Verardi \& De Marco, 2011a), até mesmo no futebol americano (Hirota, Gomes, De Marco \& Verardi, 2011b) é apresentado estabilidade no calculo do Coeficiente Alfa de ambas as orientações. 
Ano XXV, n 40, junho/2013

Aprofundando na discussão dos resultados estabelecemos a contagem das médias, desvio padrão e mediana das diferentes orientações de meta tarefa e meta ego, seguindo mais uma etapa dos objetivos.

Tabela 03: Média, Desvio Padrão e Mediana da Orientação Para Tarefa e Ego da Escola de Esporte

\begin{tabular}{|c|c|c|c|c|c|c|}
\hline \multirow{3}{*}{$\begin{array}{l}\text { Escola } \\
\text { de } \\
\text { esporte }\end{array}$} & \multirow{3}{*}{$\mathbf{n}$} & \multicolumn{4}{|c|}{ Orientação } & \multirow{3}{*}{$\begin{array}{c}\text { Mann-Whitney } \\
\text { "p" }\end{array}$} \\
\hline & & \multicolumn{2}{|c|}{ ego } & \multicolumn{2}{|c|}{ tarefa } & \\
\hline & & média & mediana & média & mediana & \\
\hline Iniciante & 37 & $2,43( \pm 1,18)$ & 2 & $4,34( \pm 1,06)$ & 5 & $0,000^{*}$ \\
\hline Básico & 89 & $2,39( \pm 1,07)$ & 2 & $4,31(+0,95)$ & 5 & $0,000^{*}$ \\
\hline Especial & 22 & $2,38( \pm 1,10)$ & 2 & $4,11(+1,06)$ & 4 & $0,000 *$ \\
\hline Total & 148 & $2,40( \pm 1,10)$ & 2 & $4,28( \pm 1,00)$ & 5 & $0,000^{*}$ \\
\hline
\end{tabular}

* Resultados significantes

Na escola de esporte, não existiu diferença significativa entre as medias de orientação para o ego, mostrando que o grupo esta homogêneo nesta característica, valor este que pode ser confirmado pela média de ego, representada pelo valor de 2 .

O mesmo resultado pode ser visto nos valores de orientação para tarefa, refletindo nos valores de mediana total de 5 (veja tabela 03). Ambos os resultados encontrado foram através do teste de anova para este grupo.

Encontramos dois (2) para meta de ego e cinco (5) para a meta de tarefa, sendo assim, de acordo com esse resultado os alunos são poucos motivados por fatores extrínsecos, e a motivação intrínseca sendo bastante presente.

Para todos os níveis de desenvolvimento foram encontradas diferenças significativas entre as orientações, mostrando que a tendência dos sujeitos da escola de esporte são de meta tarefa.

Vale lembrar que este tipo de orientação remete a sujeitos que apresentam características de serem mais persistentes, são mais otimistas, buscam a satisfação na realização de uma tarefa e demonstram segurança em seu comportamento (Winsterstein, 2002). 
Tabela 04: Média, Desvio Padrão e Mediana da Orientação Para Tarefa e Ego da ONG

\begin{tabular}{l|c|c|c|c|c|c}
\hline \multirow{2}{*}{ ONG } & \multirow{2}{*}{$\mathbf{n}$} & \multicolumn{4}{|c|}{ Orientação } & Mann-Whitney \\
\cline { 3 - 7 } & & \multicolumn{2}{|c|}{ ego } & \multicolumn{2}{c}{ tarefa } & \\
\cline { 3 - 7 } & & média & mediana & média & mediana & "p" \\
\hline Iniciante & 7 & $3,09( \pm 1,22)$ & 3 & $4,49( \pm 0,76)$ & 5 & $0,000^{*}$ \\
\hline Básico & 32 & $2,85(\underline{ \pm 1}, 21)$ & 3 & $4,39( \pm 0,86)$ & 5 & $0,000^{*}$ \\
\hline Especial & 9 & $2,31(\underline{ \pm}+1,31)$ & 2 & $4,34( \pm 1,03)$ & 5 & $0,000^{*}$ \\
\hline Total & 48 & $2,75( \pm 1,24)$ & 2 & $4,40( \pm 0,88)$ & 5 & $0,000^{*}$ \\
\hline
\end{tabular}

*Resultados significantes

Já na ONG, não existiu diferença significativa entre as medias de orientação para o ego e tarefa. O mesmo resultado foi encontrado no teste de anova para este grupo, na orientação de tarefa.

Mais uma vez estes valores podem ser verificados com as médias, destacadas na tabela 04 , com resultado total de 2 para meta de ego e 5 para meta de tarefa.

Contudo obtivemos as médias das respostas total do grupo de 4,40 para meta tarefa e 2,75 para meta ego, respectivamente, resultado estatisticamente diferente.

Em todos os níveis de desenvolvimento foram encontradas diferenças significativas entre tarefa e ego, sendo assim, mais uma vez, foi evidenciada a tendência do grupo, agora da ONG, em apresentar caraterísticas relacionadas a meta tarefa. Ou seja são indivíduos que são mais criativos e inovadores, possuem maior auto controle e acreditam em seu esforço (Winterstein, 2002).

A única diferença significativa, estatisticamente encontrada, entre as idades da ONG foi entre o nivel iniciante o nivel especial de desenvolvimento em relação a orientação para o ego, mostrado pelo teste de anova com valor de " $p$ " de 0,007.

Portanto podemos dizer que os sujeitos da ONG do nivel iniciante na meta de ego apresentam uma motivação extrínseca aflorada, sendo assim, justificamos esta postura primeiro pela idade ainda baixa, pois este nivel encontra-se entre 7 e 9 anos, e suas habilidades estão em fase de formação esportiva. Como relata Winterstein (2002) estes sujeitos são mais ansiosos, são inseguros em seu comportamento e podem ser menos persistentes.

Os valores obtidos na ONG, no sexo masculino, na orientação para tarefa no nivel iniciante foi de $4,49( \pm 0,76)$, nivel básico foi de $4,39( \pm 0,79)$ e no nivel especial a média de tarefa ficou em $4,17( \pm 0,17)$.

Na orientação para ego nivel o iniciante foi de 3,09 $( \pm 1,22)$ nivel básico foi de $3,02( \pm 1,18)$ e no nivel especial a média de tarefa ficou em 2,60 $(+1,47)$.

A mediana do sexo masculino na orientação para tarefa foi de 5, valor este representado em rodos os níveis de desenvolvimento, enquanto para ego a mediana foi de 2, também foi encontrado o mesmo valor nos níveis iniciante, básico e especial. 
Referente as alunas, do sexo feminino, da ONG os resultados de orientação para meta tarefa foi de $4,39(+1,03)$ e orientação para ego de 2,33 $(+1,19)$, valores este significativamente diferentes. No nivel especial a media de tarefa foi de 4,57 $(+0,79)$ e de ego de $1,95(+0,99)$, também estatisticamente diferentes.

A mediana do sexo feminino na orientação para tarefa foi de 5 , valor este representado em rodos os níveis de desenvolvimento, enquanto para ego a mediana foi de 2, também foi encontrado o mesmo valor nos níveis básico e especial.

Estabelecendo uma correlação entre o nível de desenvolvimento básico, comparando a orientação para ego entre os sexos, através do teste de Mann Whitney, podemos verificar uma diferença significativa de " $p$ " de 0,001 , ou seja, a média referente à orientação para o ego do sexo masculino é significativamente maior do que o sexo feminino, mostrando eu os meninos se aproximam mais das características relacionadas à meta de ego deste nível de desenvolvimento

No nível especial, referente à orientação para o ego, esta diferença não foi revelada estatisticamente (" $p$ " de 0,133 ).

Comparando os resultados de orientação para tarefa, no nível especial, através do teste de Mann Whitney, não houve diferença significativa (" $p$ " de 0,354 ), e o mesmo resultado, não significante (" $p$ " de $0,173)$, foi encontrado para a orientação para tarefa no nível especial.

Winterstein (2002) relata que as características dos indivíduos orientados para tarefa obtêm um sentimento de sucesso na realização de uma atividade proposta dependendo do eu, ou seja, de si mesmo, aonde o importante é a aprendizagem e não a performance, possuindo uma auto referência em relação ás habilidade; e atribuindo-se o sucesso á equipe, aonde o sucesso se alia ao esforço, determinação, compromisso social fazendo uso das habilidades; o esporte não é uma via de status e sim de uma boa concentração e atenção, onde o fracasso é considerado falta de esforço e determinação aonde o erro faz parte da aprendizagem.

Knijnik, Greguol, e Santos (2001) nos mostra que há uma relação entre motivação e desempenho esportivo apontando uma alternância de resultados com relação à motivação, quando esta é excessiva ou nula quando o desempenho é indesejável, entretanto quando a motivação é media o desempenho é o desejado.

Podemos lembrar que para os alunos mais novos as causas de adesão são fatores extrínseco onde recebem apoio dos familiares, o sucesso de jogadores e o status das mídias sociais; já para os adolescentes o fator intrínseco se eleva pois está voltado para melhora das habilidades motoras e da resistência física.

Contudo por mais que as crianças e adolescentes estejam com as suas motivações voltadas para o aprendizado ou sucesso, um dos papeis mais importantes para esse desenvolvimento é a participação dos professores e técnicos, aonde a motivação deve estar sempre interligada entre os participantes, visando sempre os tipos de metodologias a serem utilizadas para uma melhor adesão e ensino aprendizagem para as crianças e adolescentes (Knijnik et al., 2001).

A quarta testagem estabelecida pelo objetivo do estudo é evidenciar se existe diferença significativa separadamente de cada orientação motivacional (Tarefa e Ego) entre os estabelecimentos esportivos: 
1. Diferença entre ego dos iniciantes nos dois estabelecimentos " $p$ " de 0,002;

2. Diferença entre ego do básico nos dois estabelecimentos " $p$ " de 0,000 ;

3. Diferença entre ego do especial nos dois estabelecimentos " $p$ " de 0,389 , não significantes.

Podemos notar que ha diferença entre a orientação para o ego com relação à comparação dos estabelecimentos, são vistas na iniciação e básico, onde há uma diferença significativa para a orientação motivacional do ego, podendo levar em consideração á essas diferenças o contexto social de cada estabelecimento, onde para a ONG existe um contexto de caracterizar indivíduos por habilidades ou até mesmo em seus desempenhos esportivos durante os treinamentos e jogos, notados pelas faixas etárias de iniciação (7 á 9 anos) e básico (10 á 13 anos), verificando que nessas faixas etárias os alunos possuem uma motivação maior para vencer ou se tornar o melhor entre os colegas.

Já comparados com a escola de esporte os alunos, possuem uma motivação para o ego mais baixa em virtude das demandas que são colocadas em seu contexto, de que para eu vencer o grupo necessita trabalhar em equipe e se ajudar ou até mesmo de um bom entendimento das questões sobre não ser o melhor e sim saber a sua importância dentro do grupo e a importância de cada um.

Estabelecendo a diferença entre a orientação de meta tarefa nos diferentes estabelecimentos podemos observar:

1. Diferença entre tarefa dos iniciantes nos dois estabelecimentos " $p$ " de 0,768 não significantes;
2. Diferença entre tarefa do básico nos dois estabelecimentos " $p$ " de 0,343 não significantes;

3. Diferença entre tarefa do especial nos dois estabelecimentos " $p$ " de 0,054 não significantes.

Observando os resultados relativos a orientação para tarefa não foram encontradas diferenças significativas entre nenhum dos estabelecimentos, bem como em nenhum dos níveis de desenvolvimento de faixas etárias, sendo assim, aferimos de que ambos os estabelecimentos proporcionam uma boa vivencia para seus alunos fazendo com que eles apreendam coisas novas e se sintam motivados a continuar praticando.

Vale lembrar que a motivação intrínsecas faz com que o individuo demonstre sua auto determinação, e busque suas metas, a fim de superar seus desafios e melhorar sua performance frente a aprendizagem alcançando as etapas dos níveis de desenvolvimento, seguidos pelo iniciante, básico e especial.

\section{CONCLUSÃO}

Depois de concluídas as coletas de dados, através do instrumento aplicado e realizada as tabulações e a discussão, alguns fatores são importantes para serem levantados em conta.

A primeira constatação foi a fidedignidade do instrumento proposto, reafirmando neste estudo a validade constatada através dos resultados de coeficiente Alfa de Cronbach.

Observando os resultados nos diferentes níveis de desenvolvimento acreditamos que os sujeitos dos diferentes níveis, 
inclusive dos iniciantes, os mesmos não apresentaram dificuldades em responder as questões propostas pela escala, sendo assim possibilitando a reprodutibilidade da escala com sujeitos das diferentes idades e diferentes locais.

Portanto o instrumento proposto para identificar a motivação para tarefa e ego em crianças e adolescentes pode ser considerado valido e fidedigno como proposto no objetivo.

Levando em conta os demais objetivos do trabalho, podemos relatar que as diferenças significativas foram encontradas em ambos os estabelecimentos, tanto para orientação motivacional de tarefa ou ego.

$\mathrm{Na}$ escola de esporte a orientação para tarefa foi maior do que para o ego assim como na, resultados corroborados com as medianas onde ambos os estabelecimentos estão mais relacionados para a meta de tarefa.

Outro fator analisado foi sobre a comparação entre ambos os estabelecimentos, encontrando diferenças significativas para os níveis de desenvolvimento iniciante e básico, onde essa diferença pode ser respondida como uma forma de contextualização dos ambientes, pois as diferenças encontradas foram em relação à orientação motivacional do ego, tendo em vista que o ego para a ONG é maior comparado com a escola de esporte. Com isso podemos relatar que os alunos da ONG são mais emotivos e querem vencer e se tornar o melhor perante o grupo, diferentemente da escola de esporte que essa motivação é vista como um todo, pois, o trabalho em grupo é visto para o sucesso de todos e não o lado individual.

Contudo podemos definir que ambos os estabelecimentos apresentam uma característica mais forte em relação á orientação para tarefa quando realizamos á media e mediana, uma vez que analisado os dados, eles foram elevados para essa motivação.

Conclui-se que as características de ambos os estabelecimentos são trabalhadas em pró de seu conjunto, aonde os alunos possuem uma orientação motivacional pensando em pró do grupo, procurando vencer junto com a equipe, e desta maneira proporcionando aos alunos a possibilidade de aprender sempre, superarem seus limites, sem a busca de resultados prematuros ou a especialização precoce, levando a difusão esportiva e o desenvolvimento global dos alunos enquanto cidadãos.

\section{REFERÊNCIAS}

CAMARGO, F. P., Hirota, V. B. \& Verardi, C. A. (2008). Orientação motivacional na aprendizagem esportiva do futsal na escola. Revista Mackenzie de Educação Física e Esporte, 7 (3), 2008, 53-62.

CHAVES, W. M. (2002). O clima motivacional nas aulas de Educação Física: uma abordagem sócio cognitivista. In Coletâneas $\mathbf{2}^{\circ}$ Congresso Científico Latino - Americano da FIEP - UNIMEP, $2^{\circ}$ Simpósio Científico Cultural em Educação Física e Esportes Brasil/ Cuba, UNIMEP, Piracicaba, Junho.

DE MARCO, A. \& Junqueira, F. C. (1995). Diferentes Tipos de Influências Sobre a Motivação de Crianças Numa Iniciação Desportiva. In Piccolo, V. L. N. (org.), Educação Física Escolar: Ser... ou Não Ter? (p. 87-103). Campinas, SP: UNICAMP. 
DUDA, J. L. (1992). Motivation In Sport Settings: A Goal Perspective Approach. In Roberts, G. C. (eds.). Motivation in Sport and exercise (p. 57-91). Illinois: Human Kinetics Books.

FREIRE, J. B. (2003). Pedagogia do Futebol. Campinas: Autores Associados.

GAYA, A. \& Cardoso, M. (1998). Os fatores motivacionais para a prática desportiva e suas relações com o sexo, idade e níveis de desempenho desportivo. Revista Perfil, 2 (2), 40-52.

GOMES, R. M., Santos, P. S. M., Hirota, V. B. \& De Marco, A. (2011). Motivação na aprendizagem esportiva do futebol: Estudos preliminares. In 4 Simpósio de Iniciação Científica, UniABC, Santo André, São Paulo.

HIROTA, V. B., Schindler, P. \& Villar, V. (2006a). Motivação em atletas universitárias do sexo feminino praticantes do futebol de campo: um estudo piloto, Revista Mackenzie de Educação Física e Esporte, 5 (especial), 135-142.

HIROTA, V. B. \& De Marco, A. (2006b). Identificação do clima motivacional em escolas públicas e particulares na aprendizagem esportiva no futebol de campo: um estudo piloto. Revista Brasileira de Educação Física e Esporte, V. 20 (Suple 05), 415.

HIROTA, V. B. \& Tragueta, V. A. (2007). Verificação do clima motivacional em atletas femininas do futsal: um estudo com o questionário de orientação para tarefa ou ego (TEOSQ). Revista Mackenzie de Educação Física e Esporte, 6 (3), 207-213.

HIROTA, V. B., De Marco, A. \& Verardi, C. E. L. (2009). Avaliação da orientação motivacional de jovens atletas no futebol de campo. Revista Mackenzie de Educação Física e Esporte, 8 (2), 35-37.

HIROTA, V. B., Verardi, C. E. L. \& De Marco, A. (2011a). Motivation in the selection process of talent in soccer. In 13 FEPSAC - European Congress of Sport Psychology (p. 42), Ilha da Madeira - Portugal.

HIROTA, V. B., Gomes, M., De Marco, A. \& Verardi, C. E. L..(2011b). Avaliação da orientação motivacional no futebol americano. Revista Pulsar, 3 (2), 59.

KNIJNIK, J. D., Greguol, M. \& Santos, S. S. (2001). Motivação no Esporte Infanto Juvenil: uma discussão sobre razões de busca e abandono da prática esportiva entre crianças e adolescentes. Revista do Instituto de Ciência da Saúde, 19(1), 7-13.

MAGILL, R. A. (1984). Aprendizagem Motora: Conceitos e Aplicações. São Paulo: Edgard Blucher LTDA.

MARCONI, M. A. \& Lakatos, E. M. (2002). Técnicas de pesquisa. São Paulo: Atlas. MURRAY, E. J. (1983). Motivação e emoção. Rio de Janeiro: Zahar Editores. NICHOLLS, J. G. (1984). Achievement motivation: conceptions of ability, subjective experience, task choice, and performance. Psychological Review, 91.

PAIM, M. C. C. (2001). Fatores motivacionais e desempenho no futebol. Revista da Educação Física/UEM, Maringá, 12(2), 73-79.

THOMAS, J. R. \& Nelson, J. K. (2002). Métodos de pesquisa em atividade física. Porto Alegre: Artmed.

VERARDI, C. E. L. (2004). Interferência dos Pais e suas Consequências na prática do Futebol na Infância e Adolescência: Um Estudo de Caso, Dissertação de 
Mestrado, FACIS, UNIMEP, Piracicaba, São Paulo.

WEINBERG, R. S. \& Gould, D. (2001).

Fundamentos da Psicologia do Esporte

e do exercício. Porto Alegre: Artmed.

Winterstein, P. J. (1992). Motivação,

Educação Física e Esportes. Revista

Paulista de Educação Física, 6 (1), 53-61.
WINTERSTEIN, P. J. (2002). A Motivação para a Atividade Física e para o Esporte. In De Rose Jr., D. (org.). Esporte e Atividade Física na Infância e na Adolescência: Uma Abordagem Multidisciplinar ( $p$. 77-87). Porto Alegre: Artmed.

\title{
EVALUATION OF MOTIVATIONAL GOALS ORIENTATION IN SOCCER
}

\begin{abstract}
This study aimed to evaluate and compare the motivational goals orientation of task and ego for students belonging to two distinct environments sports football, a Private School of Sport and Non-Governmental Organization (ONG), both located in the city of Osasco - SP. Through a descriptive research we applied a scale TEOSQ to a sample of 196 subjects of both sexes aged between 7 and 17 years (mean $11.16 \pm 2.09$ ) and the data were treated with the calculation of Alpha's coefficient, mean, standard deviation, median, and the averages compared with the Mann-Whitney test. The results showed stability in the instrument with alpha of 0.78 and 0.68 for the task of self in school's sport with an average of 4.28 of task and 2.40 of ego, since the ONG's Alpha task was 0.74 and 0.60 of Alpha's ego with an average of task and ego of 4.40 and 2.75 respectively. We conclude the tendency for task-oriented with students predisposed to overeat constantly, and reliability of the instrument
\end{abstract}

Keywords: Evaluation; Soccer; Motivation; Goals Orientation.

Recebido em: novembro/2012 Aprovado em: abril/2013 